\title{
The Electrochemical Performance of Diamond Thin-Film Electrodes from Different Commercial Sources
}

\author{
Anne E. Fischer, Yoshiyuki Show, and Greg M. Swain \\ Department of Chemistry and Fraunhofer Center for Coatings and Laser Applications \\ Michigan State University \\ East Lansing, MI 48824
}

\section{Supplemental Information}

This manuscript reports on the electrochemical response of diamond electrodes from different sources, and the following text and figures are material characterization data for two commercial (Condias and Sumitomo) and two diamond electrodes produced in our laboratory (MSUMicro and MSUNano). Specifically, scanning electron micrographs and Raman spectra are presented for these diamond films.

Figure 1A-D presents scanning electron micrographs for (A) MSUMicro, (B) Condias, (C) Sumitomo, and (D) MSUNano boron-doped diamond thin-film electrodes. The crystallites in the MSUMicro, Condias and Sumitomo films possess significant triangular $(\{111\})$ faceting. There is an abundance of multiply twinned crystals (e.g., decahedral shapes with five-fold symmetry). Twins are a common defect in diamond films, particularly ones with a more octahedral morpohology, and they result when two or more intergrown crystals form in a symmetric fashion. The Condias film has a large number of re-entrant grooves and stacking faults. The re-entrant grooves, or corners, occur where two facets intersect. The re-entrant corners are sites of high growth rate. Due to a higher coordination number, chemisorbed growth precursors (e.g., methyl radicals) in the grooves are more stable against $\mathrm{H}$ attack than moieties attached to the more-open, planar surface. This gives rise to higher growth rates. 
Figure 1.

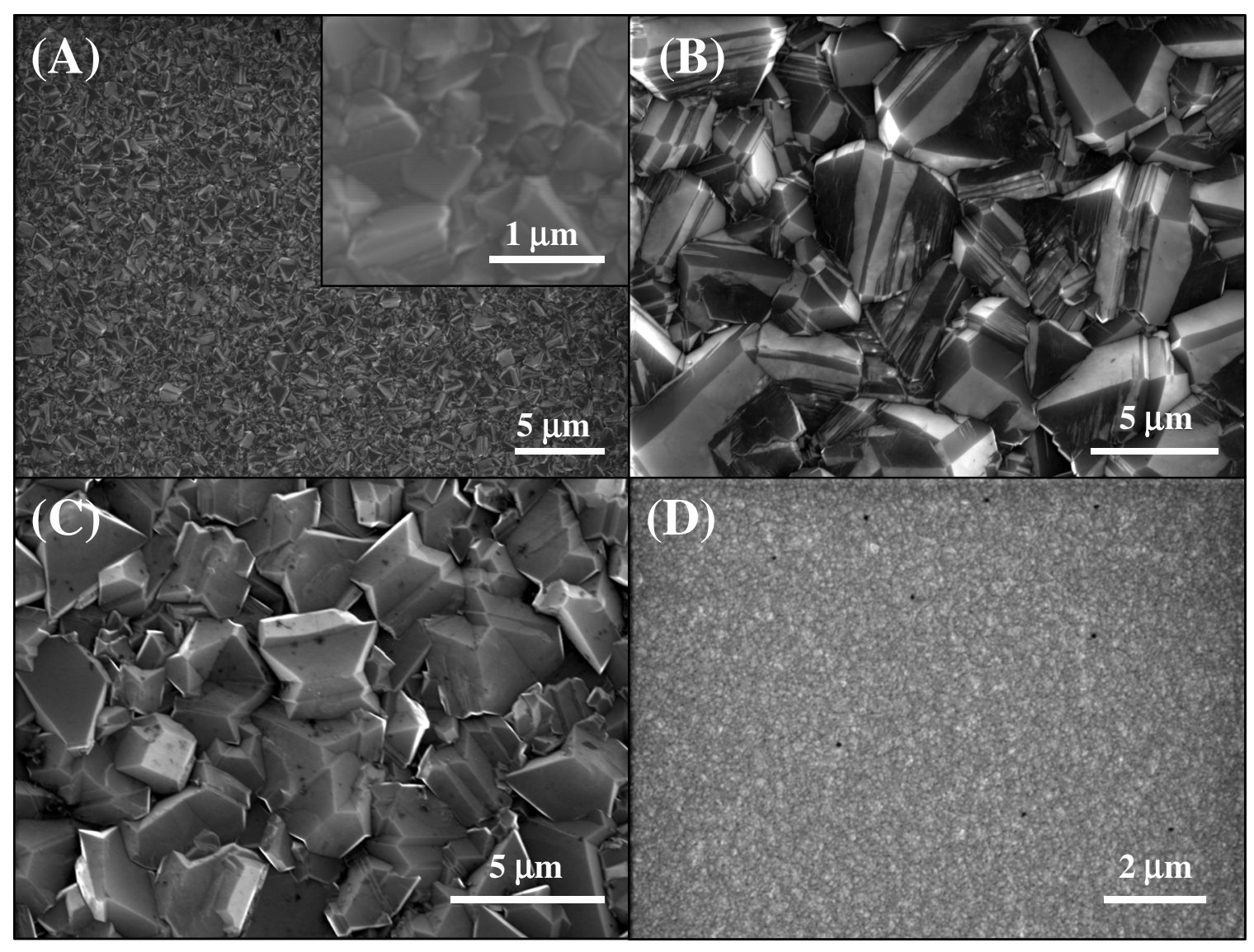


Figure 2 shows Raman spectra for the four different boron-doped diamond thinfilm electrodes. The Results and Discussion section describes some aspects of the spectra. The spectra for the Sumitomo and MSUMicro diamond electrodes, in particular, are characteristic of heavily boron-doped films. The attenuated and down-shifted diamond line, and the emergence of peaks near 500 and $1200 \mathrm{~cm}^{-1}$ are characteristic of heavily doped films (>1 x $\left.10^{20} \mathrm{~cm}^{-3}\right)$ (33-35). Pruvost et al. have observed a progressive shift toward lower wavenumbers of the one-phonon diamond line with increasing boron incorporation (36). The authors observed a downward shift of the $500 \mathrm{~cm}^{-1}$ peak with increasing doping level, and that the $1200 \mathrm{~cm}^{-1}$ peak remained practically unchanged. A transition in the spectral features representative of moderately and heavily doped diamond tends to occur in the low $10^{20} \mathrm{~B} / \mathrm{cm}^{3}$ range. For the most part, the 500 and 1200 $\mathrm{cm}^{-1}$ peaks are seen only for heavily boron-doped films, however, their presence has been reported for undoped films, as well. This makes it difficult to unequivocally ascribe them to boron-doping effects (36 and refs. therein). Gonon et al. attributed these two bands to maxima in the phonon density of states arising from scattering by phonons outside the center of the Brillouin zone (33). A breakdown of the $\mathrm{q}=0$ selection rule is associated with the loss of long range periodicity within the lattice. The local disorder results from amorphous $\mathrm{sp}^{2} / \mathrm{sp}^{3}$ bonded carbon or crystalline regions of very small size. For example, boron clusters might form at the interstitial sites and cause lattice disruption. If amorphous carbon is formed, then one would expect to see scattering intensity in the $1500-1600 \mathrm{~cm}^{-1}$ region, particularly, with visible excitation $(514.4 \mathrm{~nm})$ as the scattering cross section for graphite (a model for the nondiamond carbon impurity) is 50 times larger than for diamond (31). Such scattering is not seen in these spectra. Ushizawa et al. 
evaluated the dependence of the $1200 \mathrm{~cm}^{-1}$ scattering intensity on the excitation frequency, $\omega$, and found that it did not follow the expected $\omega^{4}$ dependence for phonon scattering (35). The authors proposed that the band might be due to electronic scattering caused by impurity (boron-related) electronic states (35). Recent Raman imaging results have revealed the presence of three additional weak bands at 610,925 , and $1045 \mathrm{~cm}^{-1}$ associated with the incorporated boron (29). The one-phonon diamond line is attenuated for the Sumitomo and MSUMicro films, because the heavier boron-doping level renders each film more opaque. The visible excitation light penetrates the film less so that the probe volume is reduced. The down-shift in the one-phonon line is correlated with the boron-doping level and may simply result from a reduced overall force constant for the lattice vibration as lighter boron atoms substitutionally insert for heavier carbon atoms within the film. 
Figure 2.

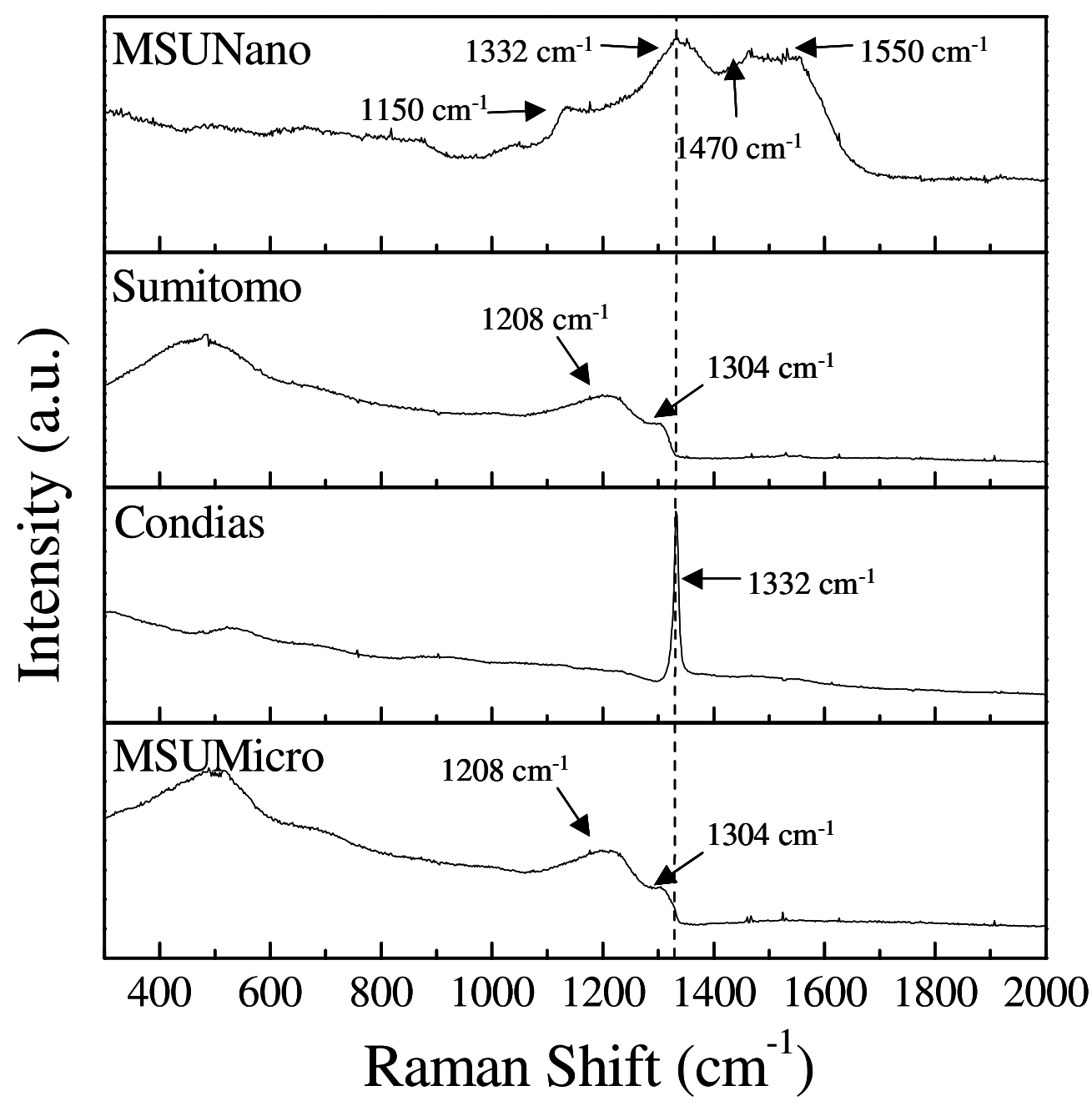

